\title{
Design and Development of Electro-brush Plating Platform Based on PLC
}

\author{
Ding Li-li
}

The First Aeronautic Institute of the Air Force, Xinyang, Henan, 464000, China

Keywords: Brush-Plating, PLC, Transducer.

Abstract. There are some disadvantages for existing electro-brush-plating platform, such as having not stepless timing, high rotate speed, and so on. We develop the brush-plating platform based on PLC and transducer. At the same time, we introduce hardware and software of the equipment respectively.

\section{Introduction}

Electro-brush plating technology is a new kind of technologies at special position of workpiece's surface which deposites metal film. It is the most means to repair and strengthen surface of the mechanical parts. The basic principle is shown in figure 1. The main equipment has the electro-brush plating platform, power supply, plating pen, and so on. It is hard to buy the electro-brush plating platform on the market at present. It usually uses general lathe instead, but there are some disadvantages, such as having not stepless timing, high speed, low degree of automation, hard to move, high cost, and so on. We develop the electro-bursh plating platform based on programmable logical controller (PLC) and transducer according to the demand analysis.

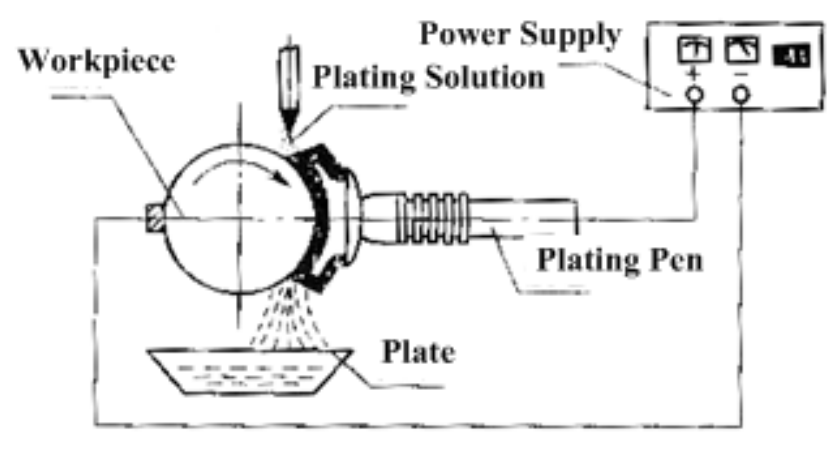

Fig.1 Principle Diagram of the Electro-brush Plating

\section{Demand Analysis and Overall Scheme Design}

According to all kinds of parts' electro-brush technology, the equipments need to control voltage, relatively speed and brush plating time. Among them, relatively speed and brush plating time is controlled by electro-brush platform. Generally its speed general requires $10 \mathrm{r} / \mathrm{min}$ to $250 \mathrm{r} / \mathrm{min}$, and its working procedure time is controlled at $3 \mathrm{~s}$ to $5 \mathrm{~min}$ during brush plating technology. So electro-brush platform's motor requires low rotation speed, wider speed range, and it can realize stepless speed regulation. Also, time controller may adopt the timer, platform requires testing and display rotation speed, and rotation speed display need four digital display meter. Moreover it can forward and reverse rotation control, set several fixed rotational speed, and display all kinds of light show.

It adopts modularization design ideas which divided into hardware design and software design in general, as shown in Fig 2. The hardware design of system includes mechanical design, motor selection, testing and display rotation speed, transducer and PLC's selection, main circuit design, PLC control circuit design, controlling panel design, and so on. The software design mainly contains PLC ladder diagram program design. 


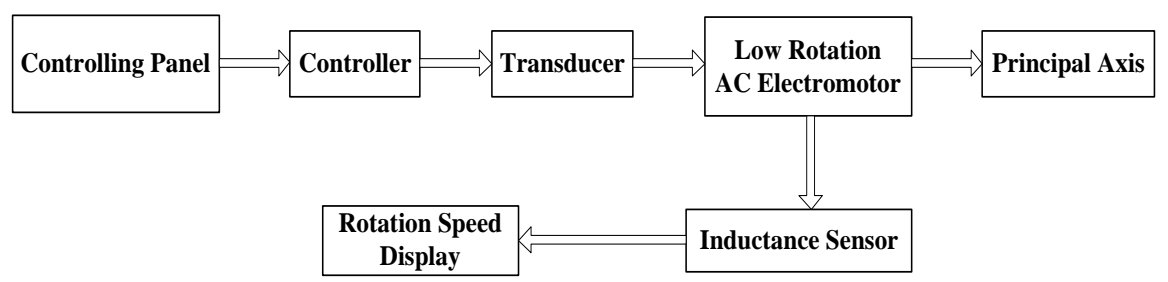

Fig.2 Composition Principle Graph of Electro-brush-plating Platform System

\section{Hardware Design}

There are many terms in the hardware system design, and we mainly introduce for motor sensor, transducer, PLC selection, design main circle, control circle and control panel’s design.

\subsection{Components Selection}

According to the type, capacity and speed of the motor, super low speed motor is adopted, and its type is YDS-100. The motor slows down by the harmonic gear deceleration which its rated power output is $1.1 \mathrm{kw}$, and rated speed is $32 \mathrm{r} / \mathrm{min}$. It can reach the required speed from frequency conversion. Speed detecting uses inductive sensor and a digital display meter SMMS-6HD which needs $12 \mathrm{~V}$ power with the range of $0 \mathrm{r} / \mathrm{min}$ to $2000 \mathrm{r} / \mathrm{min}$. The basic principle of the selecting inverter capacity is the load current does not exceed the rated current. Therefore, the system uses $0.4 \mathrm{~kW}$ to $5.5 \mathrm{~kW}$ inverter for VFD015M43B type with $230 \mathrm{~V}$ series. The core component of the system is PLC which will have a direct impact on the operability and stability of control systems. According to the functional requirements of the system and the I /O points, select C32PLC of Panasonic FP0 series with transistor output type, which has sixteen-points input and sixteen-points output with saving 10\% 15\% allowance in order to extend.

\subsection{Main Circuit Design}

The main circuit consists of power input, power switch, magnetic contactors, filters, converters, motors and other components, as shown in Fig3. The braking resistor is used to shorten motor's deceleration time, EMI filter is used to reduce the electromagnetic interference. It will select an appropriate no-fuse breaker or fuse according to the input value of the maximum current when the power is turned on. VFD015M43B inverter's input current is 4.0A, so chooses no fuse switch to $5 \mathrm{~A}$.

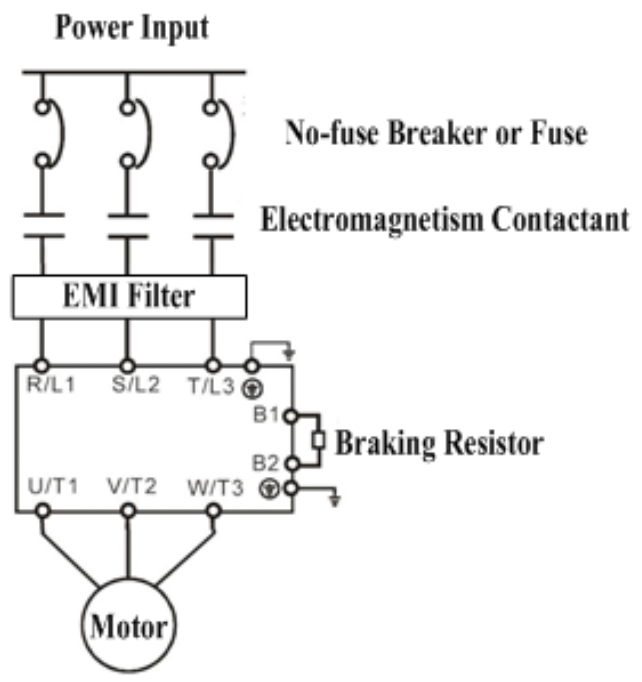

Fig.3 Main Circuit Principle Drawing 


\subsection{Control Circuit Design}

Depending on analysis I/O signal, selecting external input devices and FP0 model features of electro-brush platform with PLC control, assign PLC I/O wiring terminals, as shown in Figure 4. Of which, M0 to M5 connects inverter control ports, as shown in Figure 5. The SB1 terminal controls positive rotation, SB2 terminal controls reverse rotation, SB3 terminal controls lighting, and SB4 terminal controls resetting. HL1 terminal controls positive rotation indicator light, HL2 terminal controls reversible lights, and KM2 shows lighting contactor.

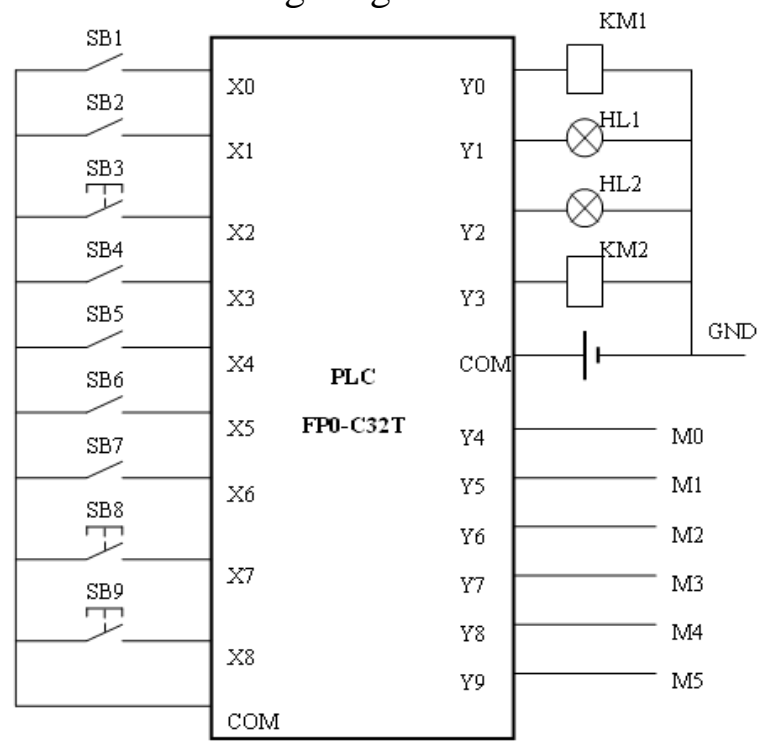

Fig. 4 Wiring PLC and Field Devices

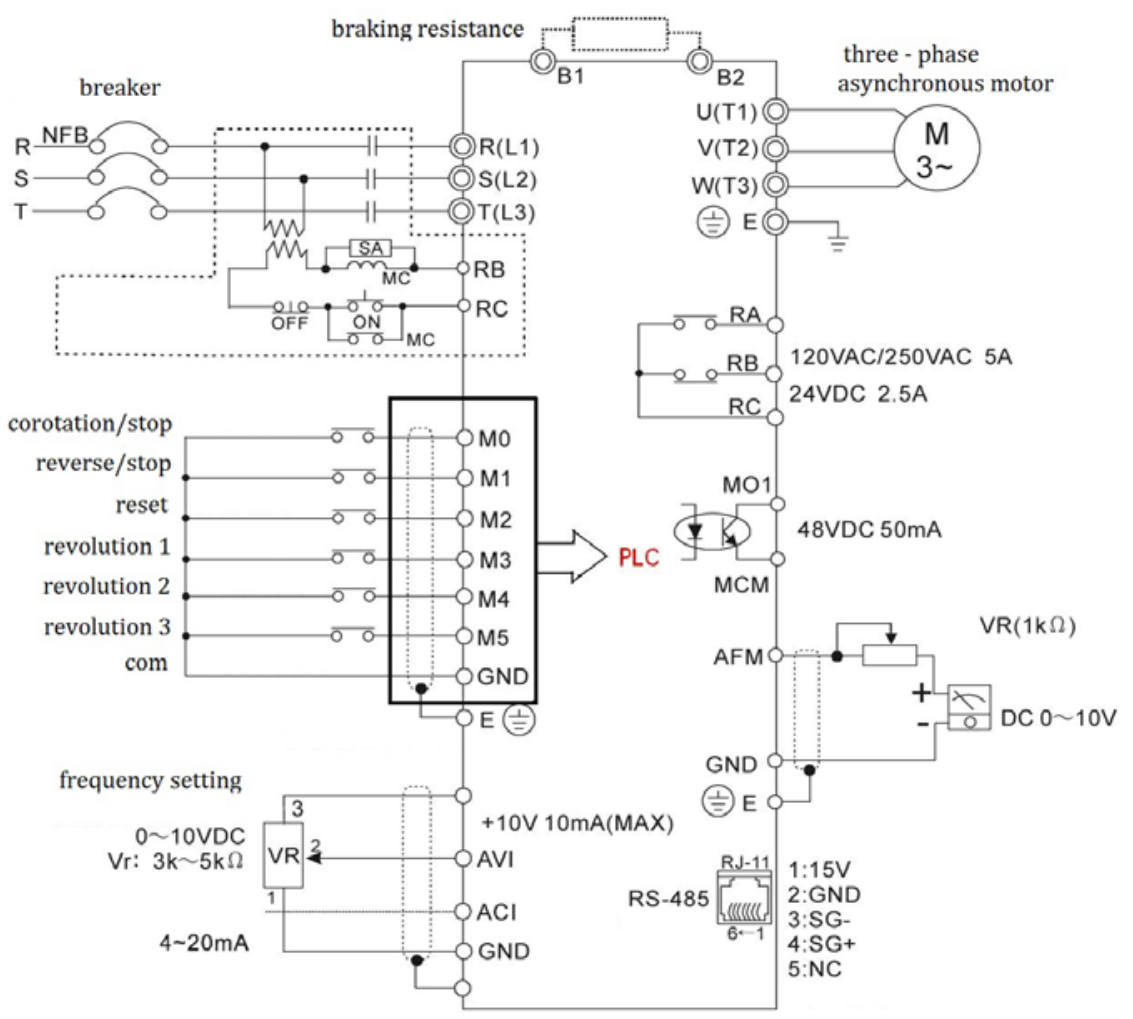

Fig. 5 Wiring Diagram of Frequency Converter and PLC

\subsection{Panel design}

Speed control panel includes a tachometer, a voltmeter, a ammeter, a power switch and four 
lights. Frequency control panel is designed with a speed display, three common frequency selection buttons and six buttons which are start button, stop button, reset button, lighting button, etc, as shown in Figure 6.
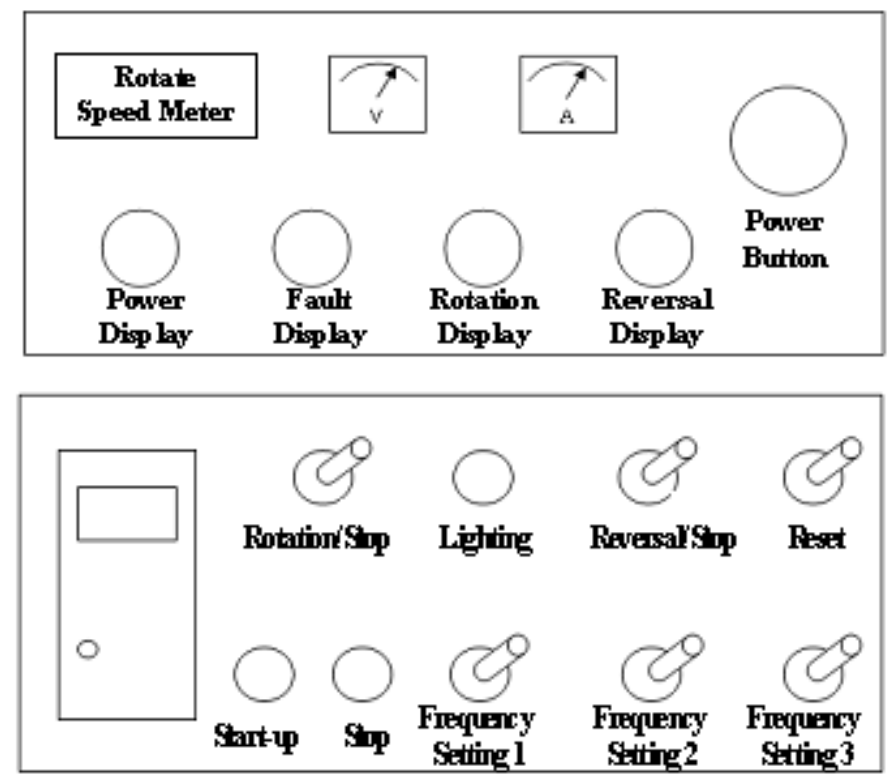

Fig. 6 Design Drawing of Control Panel

\section{Software Design}

The software design is primarily ladder program. First compile comparison table between field components and the internal PLC relay control, as shown in Table 1, and the program design shown in Figure 7. R0 is the PLC internal relay only for internal procedures to calculate, ON or OFF state does not produce an external output. Then complete writing the program on the FPWIN-GR programming platform. At last, the program will run properly and save the program through software debugging and PLC-line debugging.

Table 1 Comparison Table between Field Components and PLC

\begin{tabular}{c|c|c|c}
\hline I/O & Appellation & Electric-sign & Address \\
\hline \multirow{7}{*}{ input } & Rotation Button & SB1 & X0 \\
\cline { 2 - 4 } & Reverse Button & SB2 & X1 \\
\cline { 2 - 4 } & Lighting Button & SB3 & X2 \\
\cline { 2 - 4 } & Reset Button & SB4 & X3 \\
\cline { 2 - 4 } & Multi Speed 1 Button & SB5 & X4 \\
\cline { 2 - 4 } & Multi Speed 2 Button & SB6 & X5 \\
\cline { 2 - 4 } & Multi Speed 3 Button & SB7 & X6 \\
\cline { 2 - 4 } & Starting Button & SB8 & X7 \\
\cline { 2 - 4 } & Stopping Button & SB9 & X8 \\
\hline \multirow{5}{*}{ outpu } & Motor Contactor & KM1 & Y0 \\
\cline { 2 - 4 } & Forward light & HL1 & Y1 \\
\cline { 2 - 4 } & Reserve light & HL2 & Y2 \\
\cline { 2 - 4 } & Light contactor & KM2 & Y3 \\
\cline { 2 - 4 } & forward/stop & M0 & Y4 \\
\cline { 2 - 4 } & reserve/stop & M1 & Y5 \\
\cline { 2 - 4 } & reset & M2 & Y6 \\
\cline { 2 - 4 } & Multi Speed 1 & M3 & Y7 \\
\cline { 2 - 4 } & multi speed 2 & M4 & Y8 \\
\cline { 2 - 4 } & multi speed 3 & M5 & Y9 \\
\hline
\end{tabular}




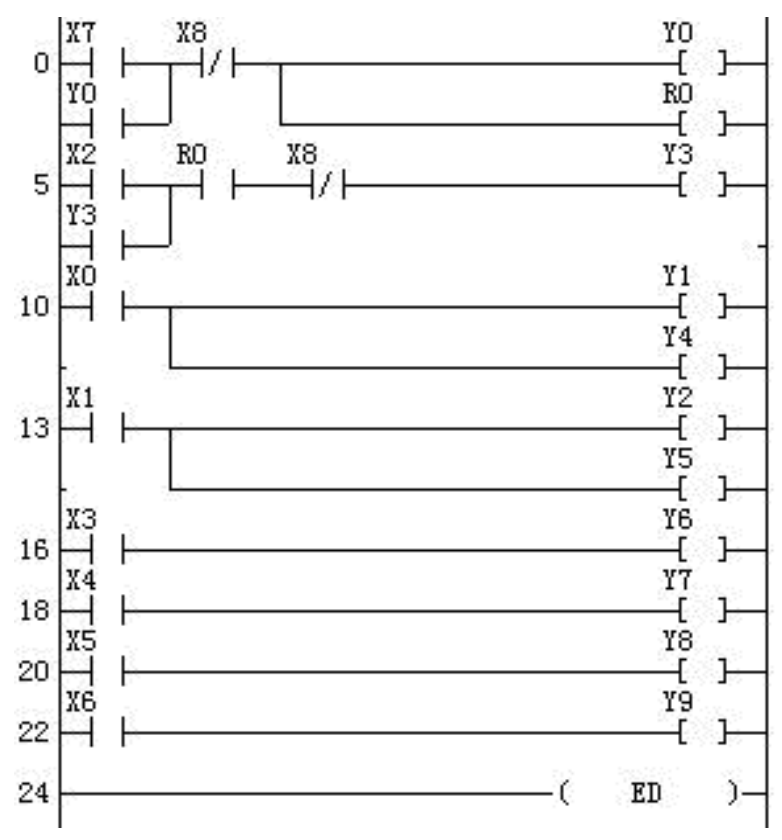

Fig.7 Ladder Diagram

\section{Summary}

After the electro-brush platform developed by more than a year of operation, it fully meets the design requirements, which achieves a wide range of variable speed in low-speed and high degree of automation with a certain application value.

\section{Reference}

[1] Li Ju, Feng Li, Zhang Zhi-qiang, Deng Yun-hua. Design of Intelligent High Voltage Pulse Generator Based on PLC. $\quad$ Automation and Instrumentation, $\quad$ 29(9), $\quad$ pp. 10-13,2014.

[2]Li Yue, Zhou Li-kun, Feng Jian-wei, Fu Gui-yong.Design of Control System for Oil Tank Cleani ng Robot Based on PLC and Electro-hydraulic Servo.Machine Tool and Hydraulics,42(9), pp. 37-40,2014.

[3] Shan Gen-li, Jiang Li-kun, Zhang Xu-jun, Liu Yao-zhen. Design of the Sling Manipulator for Deposited Cloth Machine Based on PLC. Machine Tool and Hydraulics, 42(9), pp. 59-60,2014.

[4] Yang Xiu-jie, Zhao pu-jun, Gan rong, Ying Bao-lai, Xiong Mao-tao, Luo Shu-qian.Design of the Control System for LNG Automobile Filling Stations.

Process Automation Instrumentation, 35(9), pp. 30-33,2014.

[5] Li Jiang-ping.Design of the Monitoring System for Acrylic Spinning Production Line. Process Automation Instrumentation, 35(9), pp. 27-29,2014.

[6] Sun L, Zhang Q C, Cao P T. Influence of solute cloud and precipitates on the spationemporal characteristics of the Portevin-Le Chatelier effect in A2024 aluminum alloys. Chin Phys B,18(8),pp.3500-3507,2009.

[7] Nalawade S A, Sundaram an M, Kishore R,et al. The infuluence of aging on the serrated yielding phenomena in a nickel-based superalloy. Scripta Mater, 59(9),pp.991-994,2008.

[8] Chang Tian-hia, Yin Pei-pei, Research on Secondary Elecondary Emission Yield Intellectualized Tesing System of Space Materials Based on Lingview and PLC. Chinese of Journal Electronics, pp.635-638,2014.

[9] Fu Shi-hua, Zhang Qing-chuan, Hu Qi, Gong Ming, Cao Peng-tao, Liu Hao-wen: The influence of temperature on the PLC effect in Al-Mg alloy. SCIENCE CHINA Technological Sciences, pp.1389-1393,2011.

[10] Kubin L P, Estrin Y. Evolution of dislocation desities and the critical conditions for the Portevin-Le Chatelier effect. Acta Metall Mater, 38(5),pp.697-708,1990. 
[11] Nalawade S A, Sundaram an M, Kishore R,et al. The infuluence of aging on the serrated yielding phenomena in a nickel-based superalloy. Scripta Mater, 59(9),pp.991-994,2008.

[12] Klose F B, Ziegenbein A, wei denmuller J, et al. Portevin-Le Chatelier effect in strain and stress controlled tensile tests. Comp Mater Sci, 26, pp:80-86, 2003.

[13] Liu Guo-hai, Wang Fu-liang, Shen Yue, Zhou Hua-wei, Jia Hong-ping: Realization of Neural Network Inverse System with PLC in Variable Frequency Speed-Regulating System. Lecture Notes in Computer Science, pp.257-266,2007. 